Проблеми математичного моделювання та теорії диференціальних рівнянь

\title{
УДК 519:876.2
}

\section{СИНТЕЗ АДЕКВАТНЫХ МАТЕМАТИЧЕСКИХ ОПИСАНИЙ}

Ю. Л. Меньшиков

Днепропетровский начиональный университет им. Олеся Гончара, Днепропетровск 49050. E-mail: Menshikov2003@list.ru

Рассматриваются алгоритмы построения математических описаний реальных процессов, которые описываются системой обыкновенных дифференциальных уравнений и которые позволяют получать адекватные результаты математического моделирования. Выделяются два основных подхода к проблеме построения таких описаний. В рамках одного из этих подходов предложено несколько алгоритмов. Для получения устойчивых результатов используются методы регуляризации А. Н. Тихонова для уравнений с неточно заданным оператором. Предложены новые задачи и алгоритмы построения адекватных математических описаний.

Ключевые слова. Адекватное описание, некорректные задачи, регуляризация, неточные операторы.

\section{1. Введение}

Ограничимся рассмотрением только динамических систем (процессов), которые описываются обыкновенными дифференциальными уравнениями. Отметим, что в данной работе используется терминология, которая немного отличается от общепринятой. Мы будем под математическим описанием динамических систем понимать дифференциальные уравнения, которые устанавливают связь между переменными состояния $\widetilde{x}=\left(\widetilde{x}_{1}, \widetilde{x}_{2}, \ldots, \widetilde{x}_{n_{1}}\right)^{T}$, динамической системы (выходами) и внешним воздействием $\widetilde{z}$ (входом), $\widetilde{z}=$ $\left(z_{1}, z_{2}, \ldots, z_{m_{1}}\right)^{T},(.)^{T}$ - операция транспонирования. Например, в случае линейной динамической системы эта связь может иметь вид [4]:

$$
\dot{\widetilde{x}}=C_{1} \widetilde{x}+D_{1} \widetilde{z}
$$

где $C_{1}, D_{1}$ есть матрицы с постоянными коэффициентами. Предположим, что внешнее воздействие $\widetilde{z}$ и часть переменных состояния $\widetilde{x}_{r_{1}+1}, \ldots, \widetilde{x}_{n_{1}}, r_{1}+1 \leq$ $n_{1}$ неизвестны. Другая часть переменных состояния в уравнении (1.1) измерена экспериментальным путем, то есть будем полагать, что уравнение наблюдения имеет вид:

$$
\widetilde{y}=F_{1} \widetilde{x},
$$

где $\widetilde{y}=\left(\widetilde{y}_{1}, \widetilde{y}_{2}, \ldots, \widetilde{y}_{l_{1}}\right)^{T}, F_{1}=\left\{f_{i k}\right\}_{i=1, k=1}^{i=l_{1}, k=r_{1}}-$ матрица с постоянными коэффициентами размером $r_{1} \times l_{1}$. Дополнительно будем полагать для простоты, что матрица $F_{1}$ является квадратичной и диагональной, т. е. $f_{i k}=0$ для $k \neq i, r_{1}=l_{1}$.

(c) Ю. Л. Меньшиков, 2009 
Определение 1. Математическое описание реального процесса для случая системы (1.1) будем называть адекватным по выбранным переменным состояния $\widetilde{x}_{j}(t), 1 \leq j \leq n_{1}$, если при выбранных ограничениях на внешние воздействия $z(t)$ и ограничения на значения переменных состояния при некоторых дополнительных равных условиях (начальных и граничных), выбранные переменные состояния математического описания будут совпадать с экспериментальными измерениями соответствующих физических характеристик реального процесса $\widetilde{x}_{j}(t), 1 \leq j \leq n_{1}$ в данной метрике с точностью экспериментальных измерений и точностью определения параметров математической модели процесса $\widetilde{x}_{j}(t), \widetilde{C}, \widetilde{D}$.

Будем рассматривать известную переменную состояния $\widetilde{x}_{j}(t)$ как два известных внутренних воздействия $d_{J} \widetilde{x}_{j}(t)$ и $-\left[d_{J} \widetilde{x}_{j}(t)\right], 1 \leq j \leq r_{1}, d_{j}-$ постоянные. Такая интерпретация переменной состояния позволяет упростить исходную систему. Будем называть такое преобразование «j-сечением» исходной системы [1]. Во многих случаях после ряда «сечений» исходная система (1.1) преобразуется в некоторую подсистему, у которой известна одна переменная состояния, например, $\widehat{x}_{1}(t)$, и известны все внешние воздействия $\widehat{z}_{k}(t), k=2, \ldots, m_{2}$, кроме, например, $\widehat{z_{1}}(t)$. Этот случай сводится к случаю, когда неизвестно только одно внешнее воздействие $\widehat{z}_{1}(t)=z(t)$, благодаря линейности подсистемы. Таким образом, полученная подсистема имеет вид:

$$
\dot{\widehat{x}}=C \widehat{x}+D z
$$

где $\widehat{x}=\left(\widehat{x}, \dot{\widehat{x}}, \ddot{\widehat{x}}, \ldots, \widehat{x}^{(n-1)}\right)^{T}, z=\left(z, \dot{z}, \ddot{z}, \ldots, z^{(m-1)}\right)^{T}, C, D-$ матрицы с постоянными коэффициентами. С использованием импульсной переходной функции можно записать равенство:

$$
A_{p} z=\int_{0}^{t} K(t-\tau) z(\tau) d \tau=u(t)=B_{1} \widetilde{x}, \quad \widetilde{x} \in X
$$

где $K(t-\tau)$-- известное ядро, $A_{p}-$ оператор определенной структуры $A_{p}: Z \rightarrow U ; B_{1}: X \rightarrow U ; X, U, Z$ - некоторые функциональные пространства. Если вернуться к старым переменным состояния, то уравнение (1.3) преобразуется к виду

$$
A_{p} z=B_{p} \widetilde{x}
$$

где $B_{p}$ - оператор, переводящий элементы $\widetilde{x} \in X$ в $U$.

Если с помощью ряда «сечений» не удается выделить подсистему (1.2) с одним внешним воздействием, тогда приведенные рассуждения теряют силу.

Если же исходная динамическая система (1.1) имеет несколько неизвестных внешних воздействий и для каждого из них удается получить подсистемы типа (1.2) с одним неизвестным внешним воздействием, то приведенные выше рассуждения сохраняют силу, однако дальнейшие алгоритмы построения адекватного математического описания существенно усложняются. 


\section{2. Постановка задачи}

Для успешного применения методов математического моделирования при исследовании динамических систем необходимо выполнить построение математического описания реального процесса, которое позволяет получать результаты математического моделирования, совпадающие с экспериментальными данными (измерениями) $[1,2,5,6]$. Такого совпадения добиваются путем синтеза «правильной» математической модели движения динамической системы и выбором «хорошей» модели внешнего воздействия на эту систему, если система открытая. Проиллюстрируем сказанное на примере динамической системы с сосредоточенными параметрами. Уравнение (1.4) будем рассматривать как основное. Будем предполагать, что исходные данные $\widetilde{x}=\left(\widetilde{x}_{1}, \widetilde{x}_{2}, \ldots, \widetilde{x}_{n_{1}}\right)^{T}$ получены экспериментальным путем с некоторой известной априори погрешностью:

$$
\left\|x_{T}-\widetilde{x}\right\|_{X} \leq \delta
$$

где $x_{T}-$ точные исходные данные. Проверка адекватности математического описания (модели динамической системы и модели внешнего воздействия) в данном случае сводится к проверке выполнения неравенства

$$
\rho_{U}\left(A_{p} z, B_{p} \widetilde{x}\right) \leq \varepsilon,
$$

где $\rho_{U}($.$) есть расстояние между элементами функционального пространства$ $U, \varepsilon-$ const, $\varepsilon>0, \varepsilon$ - требуемая точность совпадения с экспериментом. Если функциональные пространства являются нормированными, тогда неравенство (2.2) может иметь вид:

$$
\left\|A_{p} z-B_{p} x_{\delta}\right\|_{U} \leq \varepsilon .
$$

Естественно, что $\varepsilon$ не может быть меньше величины $\delta$. Характерной чертой для рассматриваемых задач является то, что оператор является компактным [1]. Очевидно, что при выполнении неравенства (2.2) оператор $A_{p}$ и функция $z$ связаны между собой. Нетрудно показать, что при фиксированном операторе $A_{p}$ в (2.2) существует бесконечно много различных функций z, которые будут удовлетворять неравенству $(2.2)[1,3]$. И наоборот, при некоторой фиксированной функции $z$ существует бесконечно много различных операторов $A_{p}$, для которых выполняется (2.2) [3].

Существует два основных подхода к проблеме построения пары (математическая модель поведения процесса и модель внешнего воздействия) $[1,2$, $4,5,6]$ :

1) по математической модели движения динамической системы выбранной априори структурой и неточными параметрами определяется такая модель внешнего воздействия, при использовании которой выполняется неравенство (2.2);

2) по априори заданной модели внешнего воздействия подбирается математическая модель поведения процесса заданной структуры, для которой результаты математического моделирования совпадают с экспериментом с точностью $\varepsilon$. 
В силу этого методы идентификации структуры математической модели имеют весьма ограниченную область применения. При выполнении конкретных расчетов следует учитывать, что операторы $A_{p}, B_{p}$ зависят от вектора параметров $p$ математической модели движения динамической системы, которые определяются приближенно с некоторой погрешностью. Таким образом, будем полагать, что для нормированных пространств $X, U, Z$ выполняются неравенства:

$$
\left\|A_{p}-A_{T}\right\|_{Z \rightarrow U} \leq h, \quad\left\|A_{p}-A_{T}\right\|_{X \rightarrow U} \leq d .
$$

где $A_{T}, B_{T}$ - точные операторы в уравнении $(1.4), h, d$ - известные величины.

\section{3. Объективные оценки величины $\varepsilon$}

Если величину $\varepsilon$ в неравенстве (2.2) выбирать волевым способом, то результаты проверки адекватности не будут субъективными. Поэтому представляет смысл конструировать алгоритмы проверки адекватности, в которых величина $\varepsilon$ определяется объективными факторами. Очевидно, что если операторы $A_{p}, B_{p}$ не будут изменяться в будущем при математическом моделировании процесса, то в качестве $\varepsilon$ можно взять величину $\left\|B_{p}\right\|_{X \rightarrow U} \delta$. Этот вывод следует из оценки

$$
\left\|A_{p} z_{T}-B_{p} x_{\delta}\right\|_{U}=\left\|B_{p} x_{T}-B_{p} x_{\delta}\right\|_{U} \leq\left\|x_{T}-x_{\delta}\right\|_{X} \leq\left\|B_{p}\right\|_{X \rightarrow U} \delta
$$

где $A_{p} z_{T}=B_{p} x_{T}$. Если учитывать погрешность операторов $A_{p}, B_{p}$, тогда в неравенстве (2.2) величину $\varepsilon$ следует выбирать по иному алгоритму. Будем предполагать, что существуют точные операторы $A_{T}, B_{T}$, удовлетворяющие неравенствам (2.4), для которых выполняется равенство $B_{T} x_{T}=u_{T}=A_{T} z_{T}$, где $z_{T}$-- точное решение уравнения (1.4). Тогда справедлива оценка

$$
\begin{aligned}
& \left\|A_{p} z_{T}-u_{\delta}\right\|_{U}=\left\|A_{T} z_{T}-A_{T} z_{T}+A_{p} z_{T}-u_{\delta}\right\|_{U} \\
& \quad \leq\left\|A_{p}-A_{T}\right\|\left\|z_{T}\right\|+\left\|A_{T} z_{T}-u_{\delta}\right\|_{U} \leq h\left\|z_{T}\right\|_{Z}+\left\|B_{T} x_{T}-u_{\delta}\right\|_{U} \leq\left\|z_{T}\right\|_{Z} \\
& \quad+\left\|B_{T} z_{T}-B_{p} z_{T}\right\|_{U}+\left\|B_{p} z_{T}-B_{p} z_{\delta}\right\|_{U} \leq h\left\|z_{T}\right\|_{Z}+d\left\|x_{T}\right\|_{X}+\left\|B_{p}\right\| \delta .
\end{aligned}
$$

Таким образом, можно принять

$$
\varepsilon=h\left\|z_{T}\right\|_{Z}+d\left\|x_{T}\right\|_{X}+\left\|B_{p}\right\| \delta .
$$

Оценка (3.2) является объективной, но слишком грубой, если учесть, что величины $h, d$ могут быть вычислены только если известны точные операторы $A_{T}, B_{T}$. Кроме того, величина $\left\|z_{T}\right\|_{Z}$ не является априори известной. Таким образом, оценка (3.2) не является конструктивной. Хотя величина $\left\|x_{T}\right\|_{X}$ легко оценивается через известные величины $\left\|x_{\delta}\right\|_{X}$ и $\delta$ :

$$
\left\|x_{T}\right\|_{X} \leq\left\|x_{T}-x_{\delta}\right\|_{X}+\left\|x_{\delta}\right\|_{X} \leq \delta+\left\|x_{\delta}\right\|_{X} .
$$




\section{4. Адекватность в нетрадиционных постановках задач}

Рассмотрим некоторые нетрадиционные задачи построения адекватного математического описания в рамках первого подхода $[1,4]$. Пусть $z_{p}$ есть решение экстремальной задачи:

$$
\Omega\left[z_{p}\right]=\inf _{z \in Q_{\delta, p}} \Omega[z]
$$

где $\Omega[z]$-- стабилизирующий квазимонотонный функционал $[3,4]$,

$$
Q_{\delta, p}=\left\{z:\left\|A_{p} z-B_{p} \widetilde{x}\right\|_{U} \leq\left\|B_{p}\right\| \delta\right\} .
$$

Очевидно, что любая функция из множества $Q_{\delta, p}$, включая $\Omega[z]$, будет удовлетворять условию адекватности $(3.1)$. В работах $[1,4]$ предложено несколько нетрадиционных постановок задач синтеза адекватного математического описания. Например, рассматривалась следующая задача определения модели внешнего воздействия в рамках первого подхода:

$$
\inf _{A_{p}, B_{p}} \inf _{z \in Q_{\delta, p}} \Omega[z]=\inf _{A_{p}, B_{p}} \Omega[z]=\Omega\left[z_{p}^{0}\right] .
$$

В этом случае оценка адекватности будет иметь вид:

$$
\inf _{A_{p}, B_{p}}\left\|A_{p} z_{p}^{0}-B_{p} \widetilde{x}\right\|_{U}=\inf _{A_{p}, B_{p}}\left\{\left\|A_{p}-A_{T}\right\|\left\|z_{p}\right\|+\left\|A_{p} z_{p}-B_{p} \widetilde{x}\right\|\right\}\left\|B^{0}\right\|,
$$

где $\left\|B^{0}\right\|=\inf _{B_{p}}\left\|B_{p}\right\|$. При этом использовалась свойство

$$
\left\|A_{p} z_{p}-B_{p} \widetilde{x}\right\|=\left\|B_{p}\right\| \delta
$$

регуляризованного решения для квазимонотонных операторов [3]. Для экстремальной задачи

$$
\sup _{A_{p}, B_{p}} \inf _{z \in Q_{\delta, p}} \Omega[z]=\sup _{A_{p}, B_{p}} \Omega\left[z_{p}\right]=\Omega\left[z_{p}^{1}\right] .
$$

оценка адекватности имеет вид (2.2) с величиной $\varepsilon$, равной

$$
\varepsilon=h\left\|z_{p}^{0}\right\|+\left\|B^{1}\right\| \delta,
$$

где $\left\|B^{1}\right\|=\sup _{B_{p}}\left\|B_{p}\right\|$. Для экстремальной задачи

$$
\inf _{A_{p}, B_{p}}\left\|A_{p} z_{p}\right\|=\left\|A_{p^{0}} z_{p^{0}}\right\|
$$

оценка адекватности имеет вид (2.2) с величиной $\varepsilon$ равной $\varepsilon=\left\|B^{0}\right\| \delta$. Для экстремальной задачи

$$
\sup _{A_{p}, B_{p}}\left\|A_{p} z_{p}\right\|=\left\|A_{p^{1}} z_{p^{1}}\right\|
$$

оценка адекватности имеет вид (2.2) с величиной $\varepsilon$ равной

$$
\varepsilon=\left\|B^{1}\right\| \delta .
$$


Таким образом, для различных алгоритмов синтеза моделей внешних воздействий, которые дают адекватные результаты математического моделирования в рамках первого подхода, существуют различные объективные оценки.

\section{5. Заключение}

В работе предложен алгоритм синтеза адекватных математических описаний для детерминированных процессов, поведение которых описывается системой обыкновенных дифференциальных уравнений. Предложено определение адекватного математического описания для данного случая. Сформулировано несколько различных постановок задач синтеза адекватного математического описания. Получены объективные оценки адекватности математического описания в рассматриваемых вариантах задач.

\section{Библиографические ссылки}

1. Менъшиков Ю. Л. О адекватности результатов математического моделирования // Ю.Л. Меньшиков // Сб. тр. конф. Моделирование-2008. - Киев : 2008. C. $119-124$.

2. Cnепашко B. C. Метод критических дисперсий как аналитический аппарат теории индуктивного моделирования // В. С. Спепашко. // Пробл. управ. и информат. -2008 . -№ 1. - С. 8-26.

3. Тихонов А. Н., Арсенин В. Я. Методы решения некорректных задач / А. Н. Тихонов, В. Я. Арсенин. - М. : Наука, 1979. - 365 с.

4. Menshikov Yu. L. Identification of Models of External Loads // Proc. of ICINCO 2007. - 2007. -Angers, France. - p. 376-379.

5. Gubarev V.F. Method of iterative identification of many-dimensional systems with inexact data. Part 1. Theoretical basises.// In: Problems of Control and Information, $2008,2,8-26$.

6. Gukov O. O. Algorithms of Алгоритми iterative identification of many-dimensional systems.// In: Proc. of XV International Conference on Automatical Control „Automatics -2008”, Sept. 23-26, 2008p.- Odessa: ONMA, 774-777.

7. Menshikov Yu. L. Algorithms of construction of adequate mathematical description of dynamic system. // Proceedings MATHMOD 09 Vienna- Full Papers CD Volume, Vienna Univ. of Technology, February, 2009, ISBM 978-3-901608-35-3. - p. 24822485 . 